\title{
The impact of increased reimbursement rates under the new cooperative medical scheme on the financial burden of tuberculosis patients
}

Yan-Jiao Xin ${ }^{1}$, Li Xiang ${ }^{1}$, Jun-Nan Jiang ${ }^{1}$, Henry Lucas ${ }^{2}$, Sheng-Lan Tang ${ }^{3,4}$ and Fei Huang ${ }^{5 *}$ (D

\begin{abstract}
Background: Tuberculosis (TB) is still a major public health problem in China. To scale up TB control, an innovative programme entitled the 'China-Gates Foundation Collaboration on TB Control in China was initiated in 2009. During the second phase of the project, a policy of increased reimbursement rates under the New Cooperative Medical Scheme (NCMS) was implemented. In this paper, we aim to explore how this reform affects the financial burden on TB patients through comparison with baseline data.

Methods: In two cross-sectional surveys, quantitative data were collected before (January 2010 to December 2012) and after (April 2014 to June 2015) the intervention in the existing NCMS routine data system. Information on all 313 TB inpatients, among which 117 inpatients in the project was collected. Qualitative data collection included 11 focus group discussions. Three main indicators, non-reimbursable expenses rate (NER), effective reimbursement rate (ERR), and out-of-pocket payment (OOP) as a percentage of per capita household income, were used to measure the impact of intervention by comprising post-intervention data with baseline data. The quantitative data were analysed by descriptive analysis and non-parametric tests (Mann-Whitney $U$ test) using SPSS 22.0, and qualitative data were subjected to thematic framework analysis using Nvivo10.
\end{abstract}

Results: The nominal reimbursement rates for inpatient care were no less than $80 \%$ for services within the package. Total inpatient expenses greatly increased, with an average growth rate of 11.3\%. For all TB inpatients, the ERR for inpatient care increased from 52 to $66 \%$. Compared with inpatients outside the project, for inpatients covered by the new policy, the ERR was higher (78\%), and OOP showed a sharper decline. In addition, their financial burden decreased significantly.

Conclusions: Although the nominal reimbursement rates for inpatient care of TB patients greatly increased under the new reimbursement policy, inpatient OOP expenditure was still a major financial problem for patients. Limited diagnosis and treatment options in county general hospitals and inadequate implementation of the new policy resulted in higher inpatient expenditures and limited reimbursement. Comprehensive control models are needed to effectively decrease the financial burden on all TB patients.

Keywords: Tuberculosis, New cooperative medical scheme, Financial burden, China

\footnotetext{
* Correspondence: huangfei@chinacdc.cn

${ }^{5}$ National Center for Tuberculosis Control and Prevention, China CDC, Beijing,

China

Full list of author information is available at the end of the article
}

(c) The Author(s). 2019 Open Access This article is distributed under the terms of the Creative Commons Attribution 4.0 International License (http://creativecommons.org/licenses/by/4.0/), which permits unrestricted use, distribution, and reproduction in any medium, provided you give appropriate credit to the original author(s) and the source, provide a link to the Creative Commons license, and indicate if changes were made. The Creative Commons Public Domain Dedication waiver (http://creativecommons.org/publicdomain/zero/1.0/) applies to the data made available in this article, unless otherwise stated. 


\section{Multilingual abstracts}

Please see Additional file 1 for translations of the abstract into the five official working languages of the United Nations.

\section{Background}

Despite concerted efforts in tuberculosis (TB) control since the 1990s, TB remains a major public health problem in China [1-3]. According to the World Health Organization (WHO) Global Tuberculosis Report 2018, China had the second largest estimated incidence in 2017, accounting for 9\% of TB cases worldwide. There is a long way to go to meet the goals of WHO's End TB Strategy and the UN's Sustainable Development Goals (SDGs) [4]. These are far less than 55 TB cases per 100000 population and a $75 \%$ reduction in TB deaths (compared with 2015) by 2025 and less than $10 \mathrm{~TB}$ cases per 100000 population and a 95\% reduction in TB deaths (compared with 2015) by 2035 [5]. Previous studies showed that one of the most common barriers to successful treatment is financial and that this can greatly hinder progress in scaling up care [6-8]. For example, a before-and-after study on the effects of a comprehensive programme for multi-drug resistant tuberculosis (MDRTB) in China showed that $15 \%$ of patients had to forgo treatment because of financial difficulties [7]. TB is not only an infectious disease but also a socio-economic problem [6]. Thus, greater financial risk protection measures as well as treatment interventions for patients are needed to achieve universal access to TB care [6].

$\mathrm{TB}$ is a poverty-related infectious disease, and a high cost of care may exclude poor patients from the health system and reduce the probability of adequate treatment and cure [9-13]. This may further aggravate the spread of tuberculosis and hinder progress in TB control. Thus, protecting TB patients from financial risk is a priority for policy makers [1]. Over past decades, the academic and policy communities in many countries have proposed and implemented various financial risk protection interventions and pro-poor strategies for TB control programmes to address the needs of the poor, especially in countries with a high TB burden [14]. However, the effectiveness of these programmes has often been limited.

For example, in India, after the implementation of the Revised National Tuberculosis Control Programme (RNTCP), TB remains a major cause of mortality and economic hardship [11]. A study on rural residents in Burkina Faso showed that, despite free tuberculosis care, $75 \%$ of interviewed patients faced catastrophic health expenditures [11]. In Rwanda, although the Global Fund against Acquired Immune Deficiency Syndrome, Tuberculosis and Malaria (GFATM)-funded projects greatly improved financial access to health care, most Rwandans still had problems affording the co-payment charged, thus hindering access to TB health services [15].
Globally, there is still a substantial economic burden on many TB patients, which does not conform to the definition of Universal Health Coverage (UHC): that all people receive the health services they need without being exposed to financial hardship [16]. It also runs counter to the goal of the End TB Strategy: that no affected family faces catastrophic costs due to tuberculosis [5].

Although essential TB care is free of charge in China, studies have identified that patients still have to pay significant costs for additional medicine and tests [17-19]. Moreover, under the fee-for-services (FFS) payment typically adopted in China, hospitals are mostly financed by service fees, which leads to perverse incentives for hospitals to offer more services, contributing to higher inpatient expenses and a rapid increase in healthcare costs $[20,21]$. Many previous studies have shown that health insurance is a useful tool to protect people from catastrophic health expenditure (CHE) and improve TB treatment completion rates [22-24]. For example, in the United States, TB control was enhanced by bringing uninsured Americans into the health care system [25]. At least one previous study has indicated that integrating the national TB control programme into health insurance schemes was an effective strategy to address current challenges in China [26].

Hence, in the context of the new round of Chinese health system reforms towards UHC, an innovative programme entitled the 'China-Gates Foundation Collaboration on TB Control in China' (China-Gates TB Project) was initiated in 2009 by the Chinese National Health and Family Planning Commission and Chinese Center for Disease Control and Prevention (China CDC), with support from the Bill and Melinda Gates Foundation. During the first phase (from 2009 to 2012), the financing model for TB services was changed from a special earmarked fund from the Ministry of Finance to funding mainly provided by health insurance schemes [17]. This proved challenging for the effective financing and delivery of TB services [17]. Thus, two major reforms have been implemented since 2014 during the second phase of the project: increasing the reimbursement rate to $70-80 \%$ for TB-related inpatient services and using the case-based payment method to reimburse TB designated hospitals to motivate hospitals to provide standardized treatments and contain costs [17]. However, the extent of any alleviation of the financial burden on TB patients during the implementation of these reforms is unknown. In this paper, we aim to evaluate (i) whether the effective reimbursement rate (ERR) was increased after the improvement in the nominal rate under the NCMS and (ii) the impacts of the new financing model on the financial burden of TB patients covered by the NCMS through comparison with the baseline data. 


\section{Methods}

\section{Study setting}

The data used for this paper were derived from evaluation studies for Phase II of the China-Gates Project. This aimed to implement a comprehensive TB/MDRTB control model that could be scaled up over time by the National TB Prevention and Control Program. The survey was conducted in the three cities where the project was implemented (Zhenjiang City, Jiangsu Province; Yichang City, Hubei Province; and Hanzhong City, Shaanxi Province), which are located in the eastern, central and western regions of China, respectively. Three counties (one from each category of high, middle and low GDP per capita) were then selected as study sites in each city. Two preconditions, required by the National Project Office, for TB patients to be included in the project were (1) having health insurance and (2) receiving treatment at a designated hospital. TB patients who did not meet the two preconditions were excluded from the project. This paper chose three counties, representing different levels of socio-economic development, in the central region as our study setting: Zhijiang (ZJ), Yidu (YD), and Wufeng (WF), all within the administrative area of Yichang City.

\section{Data collection}

\section{Quantitative data collection}

Data were collected by a combination of quantitative and qualitative methods. The focus was on quantitative data with some additional material provided from focus group discussions (FGDs) and document review. Quantitative data were derived from the NCMS. To understand the health expenditures and reimbursements of TB patients, we collected data before (January 2010 to December 2012) and after (April 2014 to June 2015) the intervention from the existing NCMS routine data system in each of the three counties. Eligible patients were identified via a TB diagnosis code. Hospitalization and reimbursement information on $313 \mathrm{~TB}$ inpatients were extracted from the NCMS inpatient database. The same information on 117 TB inpatients included in the project, according to the two preconditions mentioned in study setting section, was also extracted from the NCMS inpatient database. To better reflect the effect of the project, the analysis of patients excluded from the project was also added in this paper for comparison with the TB inpatients inside the project. The reimbursement data included the patient's name, sex, age group, choice of health providers, hospitalization costs and reimbursement.

\section{Qualitative data collection}

Qualitative information was used to gain a deeper and more complete understanding of the situation. Data collection included FGDs with key stakeholders, including health care providers from the designated hospitals and TB patients. The topic outlines for the FGDs were developed by the principle researchers of the investigation team, and they were conducted by senior academics with rich qualitative research experience. As shown in Table 1, approximately six participants were included in each of 11 FGDs, conducted with the help of local Centers for Disease Control and Prevention (CDC) officials. All were recorded with the permission of the participants. Policy documents relating to the reimbursement of TB patients were also collected from the study sites for review and analysis (Table 1).

\section{Data analysis \\ Quantitative data analysis}

The quantitative data were analysed using SPSS (version 22.0 Statistical software, International Business Machines Corporation, New York City, US). The main analysis focused on the expenditure and reimbursement for TB-related services and the change in effective reimbursement rates between the baseline and the intervention period. In this paper, we defined the actual implementation rate of the new policy as the number of TB inpatients reimbursed by the new policy as a percentage of total TB inpatients. Total medical expenditure was defined as the sum of reimbursement and any OOP payments. OOP payments were composed of the expenses co-paid by patients and the non-reimbursable amount that exceeded the NCMS benefit packages. The effective reimbursement rate (ERR) was defined as the actual reimbursement amount divided by the total medical expenditure [27]. The non-reimbursable expenses rate (NER) was defined as the non-reimbursable amount beyond the NCMS benefit packages divided by the

Table 1 Focus group discussions in Yichang City

\begin{tabular}{lll}
\hline FGDs & Participants & Main content \\
\hline $\begin{array}{l}\text { Health care } \\
\text { providers }\end{array}$ & $\begin{array}{l}\text { Senior administrators from TB or infectious disease section, health } \\
\text { insurance section, and hospital information section; one doctor } \\
\text { and one nurse responsible for TB care. }\end{array}$ & $\begin{array}{l}\text { Issues included the financing and expenditure of TB care, provision } \\
\text { of TB services, views and suggestions on patient diagnosis and } \\
\text { treatment management process and inter-departmental cooperation } \\
\text { mechanism }\end{array}$ \\
$\begin{array}{lll}\text { TB patients } \\
\text { Six patients who had completed a treatment course and could } \\
\text { clearly express their thoughts. Sex, age group, geographic location }\end{array}$ & $\begin{array}{l}\text { Questions regarding their diagnosis and treatment, expenses and } \\
\text { reimbursement related to TB care, and their subjective feelings } \\
\text { about their financial burden and the impacts of the project on their } \\
\text { financial burden. }\end{array}$
\end{tabular}


total medical expenditure. The nominal reimbursement rate was stipulated in the project under the NCMS. We used the ratio of OOP to the per capita income of rural residents to measure the financial burden of TB patients.

A descriptive analysis of the average medical expenses, OOP, NER, ERR and OOP/per capita income of rural residents was performed. To assess the impact of the project on the financial burden of TB patients, nonparametric tests (Mann-Whitney $U$ test) were employed to identify differences in effective reimbursement rates and the ratio of OOP to per capita income of rural residents in the baseline and intervention periodperiods. We compared the financial burden of all TB patients and TB patients included in the project in the intervention to those in the baseline in three counties. $P<0.05$ was considered statistically significant.

\section{Qualitative data analysis}

The qualitative data from the FGDs were subjected to thematic framework analysis using.

NVivo (version 10, 0 QSR International, Melbourne, Australia). First, the theme of each level initially according to the interview outline. Then, developing, a topic-stakeholders table was developed, and encoded the contents of the text information were encoded into the corresponding cells of the table to present the diverse views of different stakeholders on the same topic. In addition, new themes were identified, and the framework was further improved. After the encoding process, the contents of the two-dimensional table were summarized, and themes were formulated. Finally, the formulated themes were checked against the coded extracts to ensure that they captured the key issues raised by the participants.

As shown in Table 2, four themes were formulated: the TB prevention and control system, the implementation of the increased reimbursement, the impact of the new policy, and regulation of that policy. These four themes were checked against the coded extracts to ensure that they captured the key issues raised by the participants.

\section{Results}

\section{Reimbursement policy for TB patients under the NCMS in} Yichang City

Using health insurance policy documents and the FGDs, we analysed the inpatient service reimbursement policy for TB patients under the NCMS at baseline and during the intervention period. At baseline, the NCMS was decentralized and administered at the county level [28]. Since TB patients must now seek treatment in higher-level hospitals, the old reimbursement policy restricted the effective reimbursement rate.

In the intervention period, the reimbursement policy for TB patients was unified at the municipal level. As shown in Table 3, nominal reimbursement rates for smear-negative inpatients and smear-positive inpatients were 80 and 90\% within the package, respectively. For the actual implementation rate of the new policy Yidu and Wufeng had relatively more effective implementation, with actual reimbursement rates higher than $70 \%$ (Table 3).

\section{TB inpatient care reimbursement under the NCMS in Yichang City}

Reimbursement for TB inpatients under the NCMS before and after intervention.

As shown in Table 4, according to the NCMS database, $313 \mathrm{~TB}$ inpatients were reimbursed in the postintervention period in Yichang City compared with 1001 at baseline. For all TB patients, the mean inpatient expenses had greatly increased, from RMB 5067 to RMB 5 638 , an average growth rate of $11.3 \%$. The effective reimbursement rate over the intervention period was $66 \%$, significantly higher than that at the baseline (52\%) (Mann-Whitney U test $z=-10.854, P<0.001$ ), while OOP and NER showed a gentle decrease, but to different degrees across the three counties. The expenses co-paid

Table 2 Thematic framework analysis using FGD data

\begin{tabular}{|c|c|}
\hline First level & Second level \\
\hline TB prevention and control system & $\begin{array}{l}\text { a. Functional transformation of relevant departments under the project } \\
\text { b. Health services provided by designated hospitals in the project } \\
\text { c. The responsibilities of the Chinese Center for Disease Control and Prevention } \\
\text { d. The responsibilities of village doctors }\end{array}$ \\
\hline The implementation of the new policy & $\begin{array}{l}\text { a. Financing policy } \\
\text { b. Payment policy } \\
\text { c. Publicity and patients' awareness of the new policy }\end{array}$ \\
\hline The impact of the new policy & $\begin{array}{l}\text { a. Impacts on total cost control for TB treatment } \\
\text { b. Impacts on patients' financial burden } \\
\text { c. Views on the new financing and payment policy }\end{array}$ \\
\hline Regulation of the implementation of the new policy & $\begin{array}{l}\text { a. Regulation of designated hospitals } \\
\text { b. Regulation of medical staff behaviour in hospitals } \\
\text { c. Regulation of village doctors }\end{array}$ \\
\hline
\end{tabular}


Table 3 New reimbursement policy for TB inpatients under the NCMS in Yichang City

\begin{tabular}{lll}
\hline Nominal reimbursement rate & & $\begin{array}{l}\text { Actual implementation rate } \\
\text { of new reimbursement policy } \\
\text { Intervention }\end{array}$ \\
\hline Baseline & Intervention & ZJ: 55\%; \\
\hline Township health centers: ZJ: 85\%, YD: 85\%, WF: 80\%. & Smear-negative patients: 80\%; Smear-positive patients: 90\% & YD: 70\%; \\
County hospitals: Z: $75 \%$, YD:65\%, WF:70\%. & & WF: 74\% \\
Municipal hospital: ZJ: 50\%-65\%, YD: 50-65\%, WF: 55-65\%. & &
\end{tabular}

Data sources: collected from policy documents from health bureaus and health insurance agencies

TB Tuberculosis, NCMS New Cooperative Medical Scheme, ZJ Zhijiang City, YD Yidu city, WF Wufeng City

by patients decreased greatly, while non-reimbursable expenses had a slight increase.

Reimbursement for TB inpatients inside and outside the project after intervention.

As shown in Tables 5, $117 \mathrm{~TB}$ inpatients were included in the project, and $196 \mathrm{~TB}$ inpatients were excluded from the project in the post-intervention period. Compared with TB patients outside the project, patients inside the project had higher inpatient expenses and ERR (Mann-Whitney $U$ test $z=-13.455, P<0.001$ ).

Figures 1 and 2 show the mean OOP and ERR for TB inpatient care in the baseline and intervention periods. Across all TB patients, the mean OOP decreased greatly compared with baseline. However, the mean OOP for TB patients outside the project showed a gentle decrease, while that for TB patients in the project showed a sharp decline. Similarly, for TB patients included in the project, the ERR was significantly higher than for patients outside the project.

\section{Impact of the NCMS on TB financial burden}

The financial burden of TB patients was expressed in terms of OOP as a percentage of per capita household income. As shown in Table 6, inpatient care expenditure remained a major financial burden for TB patients. For all TB inpatients, the financial burden decreased in all three counties but differed greatly, with Wufeng having the heaviest burden (15\%). The financial burden for patients in the project significantly decreased (Mann-Whitney $\mathrm{U}$ test $z=-11.297, P<0.001$ ) and was substantially lower than that for patients excludedfrom the project.

\section{Discussion}

In this study, we found that the nominal reimbursement rates for inpatient care of TB patients greatly increased to $80-90 \%$, as shown in Table 3, under the new reimbursement policy in the project. However, for inpatient care, although the ERR for TB patients included in the project increased significantly, the impact of the new policy on all TB inpatients and TB inpatients outside the project was limited. Inpatient OOP expenditure for $\mathrm{TB}$ care remains a major financial problem for TB patients.

Although the new policy greatly improved the ERR, there was still a gap between the nominal and effective reimbursement rates. For inpatient care, the ERR for patients included in the project was significantly higher than post-intervention, and after reimbursement from the NCMS, OOP payments significantly decreased. However, for all TB patients the ERR only marginally increased, OOP payments were considerably higher in the intervention period. The reduced ERRs remain financial barriers to health services, as TB is a poverty-related disease [29].

The limited effect of the intervention on the totality of TB patients could be attributed to a number of factors.

Table 4 Reimbursement for TB inpatients under the NCMS before and after intervention

\begin{tabular}{|c|c|c|c|c|c|c|c|c|}
\hline \multirow[t]{2}{*}{ Residence } & \multirow[t]{2}{*}{ Term } & \multirow[t]{2}{*}{ Number } & \multirow{2}{*}{$\begin{array}{l}\text { Mean inpatient } \\
\text { expenses } \\
\text { (RMB) }\end{array}$} & \multicolumn{3}{|c|}{ OOP (RMB) } & \multirow{2}{*}{$\begin{array}{l}\text { NER } \\
(\%)\end{array}$} & \multirow{2}{*}{$\begin{array}{l}\text { ERR } \\
(\%)\end{array}$} \\
\hline & & & & Total & Co-paid & Non-reimbursable & & \\
\hline \multirow[t]{2}{*}{ ZJ } & Baseline & 242 & 6372 & 3048 & 2220 & 828 & 13 & 52 \\
\hline & Intervention & 76 & 6231 & 2034 & 1286 & 748 & 12 & $67^{* * *}$ \\
\hline \multirow[t]{2}{*}{ YD } & Baseline & 430 & 4522 & 2397 & 2035 & 362 & 8 & 47 \\
\hline & Intervention & 150 & 5508 & 2135 & 1639 & 496 & 9 & $61^{* * *}$ \\
\hline \multirow[t]{2}{*}{ WF } & Baseline & 329 & 4818 & 2076 & 1787 & 289 & 6 & 57 \\
\hline & Intervention & 87 & 5344 & 1495 & 1281 & 214 & 4 & $72^{* * *}$ \\
\hline \multirow[t]{2}{*}{ Total } & Baseline & 1001 & 5067 & 2450 & 1994 & 456 & 9 & 52 \\
\hline & Intervention & 313 & 5638 & 1933 & 1454 & 479 & 8 & $66^{* * *}$ \\
\hline
\end{tabular}

Data source: NCMS database

TB Tuberculosis, NCMS New Cooperative Medical Scheme, ZJ Zhijiang City, YD Yidu City, WF Wufeng City, OOP Out-of-pocket payment, NER Non-reimbursable expenses rate, ERR Effective reimbursement rate

:*** significant at the $1 \%$ levels 
Table 5 Reimbursement for TB inpatients inside and outside the project after intervention

\begin{tabular}{|c|c|c|c|c|c|}
\hline Residence & Patients & Number & Mean inpatient expenses (RMB) & $\mathrm{OOP}(\mathrm{RMB})$ & ERR (\%) \\
\hline \multirow[t]{2}{*}{ ZJ } & Intervention-a & 41 & 4859 & 917 & 81 \\
\hline & Intervention-b & 35 & 7838 & 3342 & $57^{* * *}$ \\
\hline \multirow[t]{2}{*}{ YD } & Intervention-a & 48 & 6602 & 1748 & 74 \\
\hline & Intervention-b & 102 & 4993 & 2317 & $53^{* * *}$ \\
\hline \multirow[t]{2}{*}{ WF } & Intervention-a & 28 & 5740 & 1064 & 81 \\
\hline & Intervention-b & 59 & 5156 & 1700 & $67^{* * *}$ \\
\hline \multirow[t]{2}{*}{ Total } & Intervention-a & 117 & 5785 & 1293 & 78 \\
\hline & Intervention-b & 196 & 5550 & 2315 & $59^{* * *}$ \\
\hline
\end{tabular}

Data source: NCMS database

Note: intervention-a indicates reimbursement data for TB patients included in the project and intervention-b reimbursement data for TB patients excluded from the project

TB Tuberculosis, ZJ Zhijiang City, YD Yidu City, WF Wufeng City, OOP Out-of-pocket payment, ERR Effective reimbursement rate

${ }^{* * *}$ : significant at the $1 \%$ levels

First, the fact that some TB patients were excluded from the project limited the effect of our project. From our results, we found that patients outside the project had higher OOP and lower ERR and that the financial burden for TB inpatients outside the project was substantially heavier than that for TB inpatients in the project post-intervention. Analysis of policy documents indicated that the two preconditions specified above excluded somesome uninsured TB patients and patients receiving treatment at non-designated hospitals from the project. Additionally, the FGDs showed that in Yichang City, some unreasonable human factors limited the range of intervention. For example, in YD, patients with complications were typically admitted under other diagnoses and were thus also excluded. Some designated hospitals excluded patients with high expenditures exceeding the quota of the case-based payment from the project. Second, comprehensive policy implementation was required. Although the three selected counties were in principle implementing the same reimbursement policy, the resulting ERRs varied considerably. Analysis suggests that low levels of reimbursement correlated with limited policy implementation. Taking Zhijiang as an example, its policy implementation rate, which was only $55 \%$, was the lowest among the three counties, and its effective reimbursement rate was only $67 \%$.

The fact that more inpatients sought medical care at municipal or higher-level hospitals may be another factor lowering the overall ERR. This not only results in higher inpatient expenditures by $\mathrm{TB}$ patients, for example, on supplementary drugs and additional tests, but can also restrict reimbursement for inpatient care because it tends to encourage a more cautious and restrictive approach by the NCMS, meaning that TB

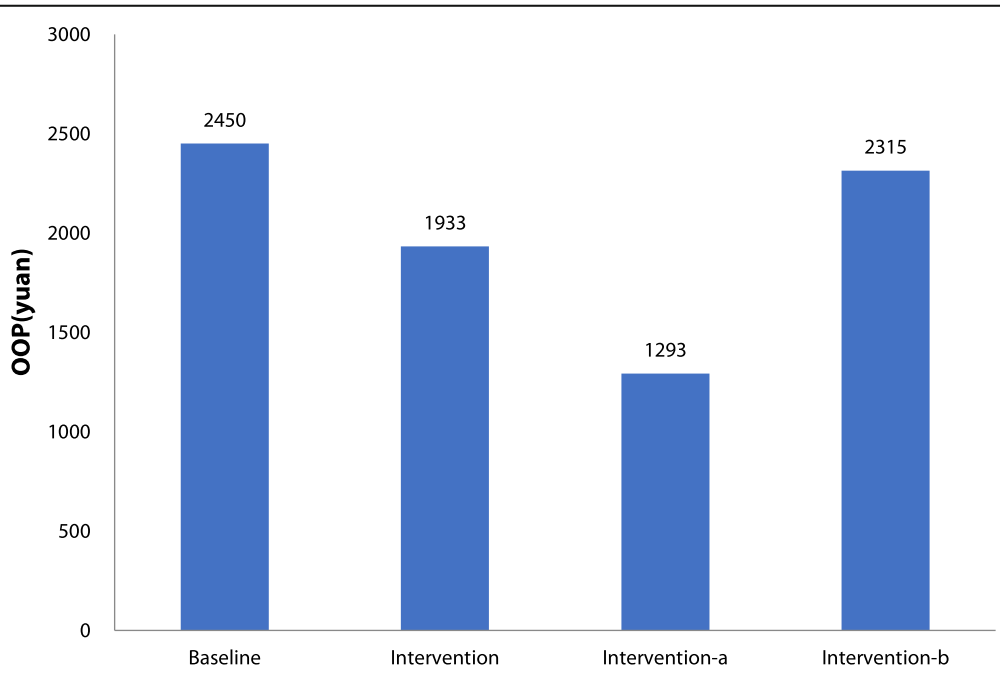

Fig. 1 Mean out-of-pocket payment for Tuberculosis inpatients before and after intervention Note: interview-a indicates reimbursement data for TB patients included in the project; Intervention-b reimbursement data for TB patients excluded from the project; OOP: out-of-pocket payment 


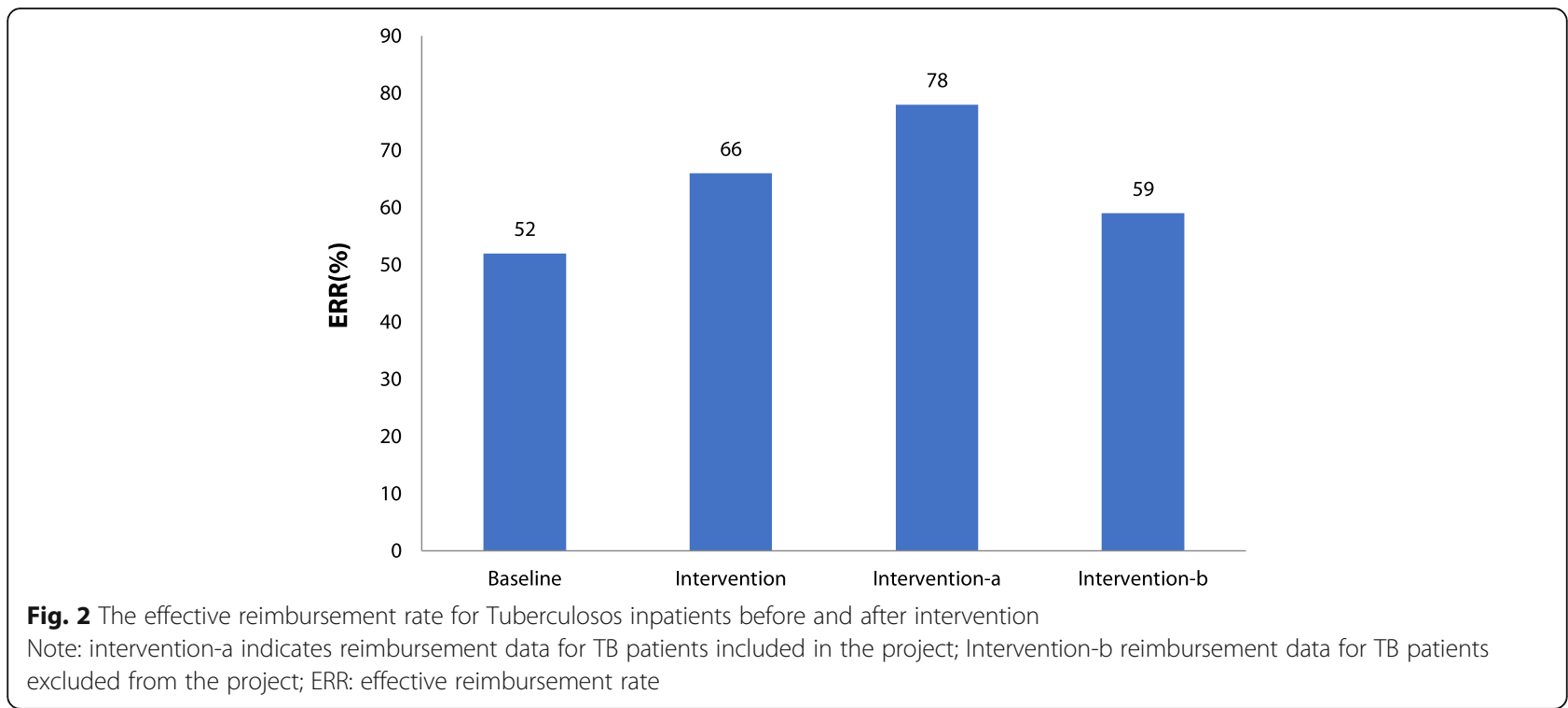

patients hospitalized at the municipal or higher-level hospitals may often have been excluded from the intervention. To some extent, the increase in the use of higher-level hospitals is probably related to a perception among patients that county general hospitals have more limited diagnosis and treatment options and less highly qualified staff.

With major improvements in infrastructure, it has become much easier for TB patients to travel to municipal or higher-level hospitals. The ever increasing use of higher-level facilities may well continue if there is no measure to control access. Comprehensive TB control models for the effective diagnosis, treatment and management of TB patients will require further efforts to enhance professional and health education programmes at the county level $[17,21,30]$. It may also be the case that county-level designated hospitals are refusing to treat patients with relatively severe illness whose treatment cost might exceed the standard for a single disease under the case-based payment mechanism. This question requires further research.
Although the ERR for TB inpatient care for those included in the project improved significantly and their OOP payment decreased substantially, OOP as a percentage of per capita household income for rural residents still often exceeded $10 \%$, usually taken as the threshold of catastrophic health expenditure [1]. For TB inpatients excluded from the project, the situation was considerably worse. The financial burden between TB inpatients inside and outside the project was remarkably different: TB inpatients outside the project had a heavier financial burden than TB inpatients in the project. This is partly a reflection of the fact that TB is a poverty-related disease, with most TB patients having low socioeconomic status. The total health expenditure post-intervention increased remarkably. One reason may be that improvement in the reimbursement rate for $\mathrm{TB}$ patients promoted an increase in the demand for TB services and treatment compliance [31]. Some studies have shown that poverty is a main factor restricting the utilization of TB health services, and failure to adhere to the complete treatment cycle of TB care still exists in many areas of China [19, 32]. Note that,

Table 6 Financial burden of TB patients before and after intervention

\begin{tabular}{|c|c|c|c|c|c|c|}
\hline \multirow[t]{2}{*}{ residence } & \multicolumn{2}{|c|}{ Mean per capita income of rural residents (RMB) } & \multicolumn{4}{|c|}{ OOP/per capita income of rural resident (\%) } \\
\hline & baseline & intervention & baseline & intervention & intervention-a & intervention-b \\
\hline ZJ & 9169 & 15285 & 33 & $13^{* * *}$ & $6^{* * *}$ & $22^{* * *}$ \\
\hline YD & 9096 & 15035 & 26 & $14^{* *}$ & $12^{* * *}$ & 15 \\
\hline WF & 3815 & 7146 & 54 & $21^{* * *}$ & $15^{* * *}$ & 24 \\
\hline Total & 7027 & 11837 & 35 & $16^{* * *}$ & $11^{* * *}$ & $20^{* *}$ \\
\hline
\end{tabular}

Data source: NCMS database

Note: intervention-a indicates reimbursement data for TB patients included in the project and intervention-b reimbursement data for TB patients excluded from the project

TB Tuberculosis, ZJ Zhijiang City, YD Yidu City, WF Wufeng City, OOP Out-of-pocket payment

${ }^{* *},{ }^{* * *}:$ significant at the 5 , and $1 \%$ levels, respectively 
as TB disproportionally affects the poor population in less-developed regions, patients may suffer an additional financial burden due to interrupted and/or inappropriate treatment [33], which will also impact non-medical costs, including travel, subsistence and loss of earnings.

Cost control is essential to reduce the cost of care and the risk of a range of serious problems, including treatment delays, poor treatment adherence and drug resistance $[1,19,34]$. It seems clear that poor implementation of the new policy weakened the intended impact on cost control. Case-based payments were the preferred option. Compared with a fee-for-services approach, this was seen as one way to reduce the provision of unnecessary services [9, 31]. However, inadequate implementation weakened any impact of cost control, contributing to the high level of OOP.

This result may have been influenced by the attitudes of some key actors. Several CDC directors suggested that it was unfair to patients with other diseases to increase reimbursement for TB alone. Limited health service packages that excluded some essential diagnosis and treatment services were another reported reason for ineffective implementation of the new policy. Officials of health insurance agencies indicated that they thought that the package reflected a high standard of treatment and emphasized that they had not been involved in its formulation. They simply implemented the policy and suggested that the design might have been improved if they had participated. However, designated hospital managers expressed the opinion that using the average cost of TB treatment over the previous 3 years as the basis for the standard package was too simplistic. The cost for a considerable number of patients with severe illness or complications substantially exceeded this estimate. Some participants argued that the revenue targets set for their hospital could not be achieved under the new policy and that the limited health service packages might constrain clinical options and cause great concern to doctors who felt they were not providing the optimal treatment. Given the negative attitudes of some health service providers, a generally agreed standard, improved monitoring and effective supervision are needed to guarantee that the policy reforms are implemented as intended.

\section{Limitations}

First, we only evaluated the quantitative implications of the project from the perspective of patients. The impact on service providers (doctors, designated hospitals, government officials, etc.) are equally important but were not analysed in the context of the new policy. Second, as we discussed, some unreasonable human factors excluded TB inpatients from the project, which may influence the conclusion. However, the percentages of patients excluded from project with different reason were unobtainable from the data we have. And our further research will focus on this point.

\section{Conclusions}

Although the nominal reimbursement rates for inpatient care of TB patients greatly increased following the intervention, there was still a gap between the nominal and effective reimbursement rates. The exclusion of many patients from the benefits of the intervention considerably reduced its impact. Inpatient OOP expenditure remained a major financial problem for patients, partly because $\mathrm{TB}$ is a poverty-related disease. Comprehensive TB control models, including enhancing professional and health education programmes at the county level, setting generally agreed standards, improving monitoring and ensuring effective supervision, are needed to guarantee that such projects are implemented as intended and effectively decrease the financial burden of all TB patients.

\section{Additional file}

Additional file 1: Multilingual abstracts in the five official working languages of the United Nations. (PDF $217 \mathrm{~kb}$ )

\section{Abbreviations}

CHE: Catastrophic health expenditure; ERR: Effective reimbursement rate; FFS: Fee for services; FGDs: Focus group discussions; MDRTB: Multi-drug resistant tuberculosis; NCMS: New Cooperative Medical Scheme; NER: Nonreimbursable expenses rate; OOP: Out-of-pocket payment; TB: Tuberculosis; UHC: Universal Health Coverage; WF: Wufeng County; YD: Yidu County; ZJ: Zhijiang County

\section{Acknowledgements}

This paper is part of the output emanating from the programme entitled "China National Health and Family Planning Commission and the Gates Foundation TB Project (Phase II)", a collaboration between the Government of China and the Melinda and Bill Gates Foundation (Grant No. 51914) and implemented by the Chinese Center of Disease Control and Prevention (China CDC). The Duke Global Health Institute, USA/Duke Kunshan University in China, Nanjing Medical University, Huazhong University of Science and Technology and Xi'an Jiaotong University in China were contracted by the Foundation and China CDC to undertake the baseline survey and the final evaluation to assess the impact of the comprehensive model of TB/MDRTB control and care in China. Dr. Xie-Xiu Wang, Dr. Shuigao Jin, Dr. Shi-Wen Jiang, the three senior advisors of the project, participated in part of the survey and provided very helpful technical support to the project, for which the authors of the paper are immensely grateful. The authors of the paper also express their sincere appreciation to Dr. Daniel Chan and Shi-Tong Huang from the Gates Foundation, You Li, Miao-Miao Sun from project management office at China CDC, and other colleagues from China CDC at the national, provincial, prefectural and county levels, as well as from TB designated hospitals at the prefectural and county levels in the three project prefectures, who generously contributed their time to the implementation of the study.

\section{Authors' contributions}

$Y X, L X, J J$ and $F H$ carried out the literature review, fieldwork, data collection, analysis and interpretation. YX drafted the initial manuscript with reviews from $L X, H L, S T$ and $F H$, who contributed to modifying the articles in detail to the final version. $\mathrm{HL}$ assisted in the interpretation of the results and revised the manuscript. ST participated in results interpretation and paper writing. All authors jointly made substantial contributions to the conception, design and acquisition of data. The final manuscript submitted for publication was read and approved by all authors.

\section{Funding}

This work was supported by the "China National Health and Family Planning Commission and the Gates Foundation TB Project (Phase II)" (Grant No. 51914) 
and the project "Study on the Dynamic Optimization of Catastrophic Health Insurance Reimbursement Modes and the Scale of Fund Expenditure in the Perspective of UHC" (Grant No. 71573095), supported by the National Natural Science Foundation of China.

\section{Availability of data and materials}

The datasets used and analysed during the current study are available from the corresponding authors upon reasonable request.

\section{Ethics approval and consent to participate}

Ethical permission was obtained from the Institutional Ethics Committee, Chinese Center for Disease Control and Prevention, China (No. 201626).

\section{Consent for publication}

Not applicable.

\section{Competing interests}

The authors declare that they have no competing interests.

\section{Author details}

'School of Medicine and Health Management, Huazhong University of Science and Technology, Wuhan, China. ${ }^{2}$ Institute of Development Studies, Brighton, UK. ${ }^{3}$ Duke Global Health Institute, Duke University, Durham, NC, USA. ${ }^{4}$ Global Health Research Center, Duke Kunshan University, Kunshan, China. ${ }^{5}$ National Center for Tuberculosis Control and Prevention, China CDC Beijing, China.

\section{Received: 8 September 2018 Accepted: 9 July 2019}

\section{Published online: 02 August 2019}

\section{References}

1. Xiang L, Pan $Y$, Hou S, Zhang H, Sato KD, Li Q, et al. The impact of the new cooperative medical scheme on financial burden of tuberculosis patients: evidence from six counties in China. Infect Dis Poverty. 2016:5:8.

2. Wang L, Liu J, Chin DP. Progress in tuberculosis control and the evolving public-health system in China. Lancet. 2007:369:691-6.

3. Wang L, Zhang H, Ruan Y, Chin DP, Xia Y, Cheng S, et al. Tuberculosis prevalence in China, 1990-2010; a longitudinal analysis of national survey data. Lancet. 2014;383:2057-64.

4. Falzon D, Jaramillo E, Wares F, Zignol M, Floyd K, Raviglione MC. Universal access to care for multidrug-resistant tuberculosis: an analysis of surveillance data. Lancet Infect Dis. 2013;13:690-7

5. WHO. Draft Report of the Sixty-Seventh World Health Assembly on Global Strategy and Targets for Preventiontuberculosis prevention, care and control after 2015: World Health Organization; 2015. https://econpapers. repec.org/RePEc:ess:wpaper:id:6861. Accessed 6 July 2018

6. Wingfield T, Boccia D, Tovar M, Gavino A, Zevallos K, Montoya R, et al. Defining catastrophic costs and comparing their importance for adverse tuberculosis outcome with multi-drug resistance: a prospective cohort study, Peru. PLoS Med. 2014;11:e1001675.

7. Li R, Ruan $Y$, Sun $Q$, Wang $X$, Chen $M$, Zhang $H$, et al. Effect of a comprehensive programme to provide universal access to care for sputum-smear-positive multidrug- resistant tuberculosis in China: a before-and-after study. Lancet Glob Health. 2015:3:e217-28.

8. Hua J, Hof SSVD, Xu L, Qiu Y, Hou J, Werf MJ. Risk factors for non-cure among new sputum smear positive tuberculosis patients treated in tuberculosis dispensaries in Yunnan, China. BMC Health Serv Res. 2011;11:97.

9. Ye J, Zhang J, Gao S, Ma Y, Liu Q. Health resource use and cost under pay on fee and case-based payment in hospitalized patients with chronic obstructive pulmonary disease in China. Value Health. 2016;19:A878. Available from https://doi.org/10.1016/j.jval.2016.08.319.

10. Barter DM, Agboola SO, Murray MB, Barnighausen T. Tuberculosis and poverty: the contribution of patient costs in sub-Saharan Africa--a systematic review. BMC Public Health. 2012;12:980.

11. Narula P, Azad S, Lio P. Bayesian melding approach to estimate the reproduction number for tuberculosis transmission in Indian states and union territories. Asia Pac J Public Health. 2015;27:723-32.

12. Méda ZC, Lin YT, Sombié I, Maré D, Morisky DE. Medication-adherence predictors among patients with tuberculosis or human immunodeficiency virus infection in Burkina Faso. J Microbiol Immunol. 2014;47:222-32.
13. Laokri S, Weil O, Drabo KM, Dembelé SM, Kafando B, Dujardin B. Removal of user fees no guarantee of universal health coverage: observations from Burkina Faso. Bull World Health Organ. 2013;91:277-82.

14. Ahn Dl. Addressing poverty in TB control. World Health Organization. 2005. https://www.who.int/tb/publications/tb-control-poverty/en/. Accessed 3 July 2018

15. Kalk A, Groos N, Karasi J, Girrbach E. Health systems strengthening through insurance subsidies: the GFATM experience in Rwanda. Tropical Med Int Health. 2010;15:94-7.

16. Pan H, Bele S, Feng Y, Qiu S, Lü J. Analysis of the economic burden of diagnosis and treatment of tuberculosis patients in rural China. Int $J$ Tuberc Lung D. 2013:17:1575-80.

17. Tang S, Wang L, Wang H, Chin DP. Access to and affordability of healthcare for TB patients in China: issues and challenges. Infect Dis Poverty. 2016;5:10.

18. Liu Q, Smith H, Wang Y, Tang S, Wang Q, Garner P. Tuberculosis patient expenditure on drugs and tests in subsidised, public services in China: a descriptive study. Tropical Med Int Health. 2010:15:26-32.

19. Long Q, Smith H, Zhang T, Tang S, Garner P. Patient medical costs for tuberculosis treatment and impact on adherence in China: a systematic review. BMC Public Health. 2011;11:393.

20. Hu S, Tang S, Liu Y, Zhao Y, Escobar ML, Ferranti D. Reform of how health care is paid for in China: challenges and opportunities. Lancet. 2008;372:1846-53.

21. Huynh $\mathrm{GH}$. Can China achieve the WHO global targets for TB control by 2035? T Roy Soc Trop Med H. 2016;110:161-2.

22. Bennett $\mathrm{S}$. The role of community-based health insurance within the health care financing system: a framework for analysis. Health Policy Plan. 2004;19:147-58.

23. Hirsch-Moverman Y, Shrestha-Kuwahara R, Bethel J, Blumberg HM, Venkatappa TK, Horsburgh CR, et al. Latent tuberculous infection in the United States and Canada: who completes treatment and why? Int J Tuberc Lung D. 2015;19:31-8.

24. Zhang L, Cheng X, Tolhurst R, Tang S, Liu X. How effectively can the new cooperative medical scheme reduce catastrophic health expenditure for the poor and non-poor in rural China? Tropical Med Int Health. 2010;15:468-75.

25. Balaban V, Marks SM, Etkind SC, Katz DJ, Higashi J. Tuberculosis elimination efforts in the United States in the era of insurance expansion and the affordable care act. Public Health Rep. 2015;130:349.

26. Tang S. Tackling challenges of TB/MDRTB in China: concerted actions are imperative. Infect Dis Poverty. 2015:4:19.

27. Yi H, Zhang L, Singer K, Rozelle S, Atlas S. Health insurance and catastrophic illness: a report on the new cooperative medical system in rural China. Health Econ. 2009:18(Suppl 2):119-27.

28. Huang F, Zhang H, Lv Q, Sato KD, Qu Y, Huan S, et al. Use of antituberculosis drugs among newly diagnosed pulmonary tuberculosis inpatients in China: a retrospective study. Infect Dis Poverty. 2016;5:2.

29. Zhou C, Long Q, Chen J, Xiang L, Li Q, Tang S, et al. Factors that determine catastrophic expenditure for tuberculosis care: a patient survey in China. Infect Dis Poverty. 2016:5:6.

30. Long Q, Qu Y, Lucas H. Drug-resistant tuberculosis control in China: progress and challenges. Infect Dis Poverty. 2016:5:9.

31. Laokri S, Drabo MK, Weil O, Kafando B, Dembele SM, Dujardin B. Patients are paying too much for tuberculosis: a direct cost-burden evaluation in Burkina Faso. PLoS One. 2013:8:e56752

32. Xu B, Fochsen G, Xiu Y, Thorson A, Kemp JR, Jiang QW. Perceptions and experiences of health care seeking and access to TB care-a qualitative study in rural Jiangsu Province, China Health Policy 2004; 69:139-149.

33. Jin PPY, Biller-Andorno N, Wild V. Ethical implications of case-based payment in China: a Analysissystematic analysis. Dev World Bioeth. 2015;15:134-42.

34. Raviglione M, Marais B, Floyd K, Lonnroth K, Getahun H, Migliori GB, et al. Scaling up interventions to achieve global tuberculosis control: progress and new developments. Lancet. 2012;379:1902-13. 